\title{
Effects of Taohongsiwu decoction on the expression of $\alpha$-SMA and TGF- $\beta 1$ mRNA in the liver tissues of a rat model of hepatic cirrhosis
}

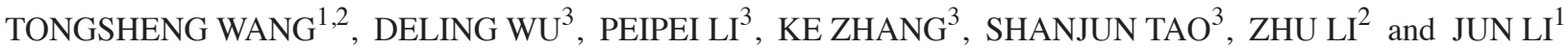 \\ ${ }^{1}$ School of Pharmacy, Anhui Medical University, Hefei, Anhui 230032; ${ }^{2}$ Department of Pharmacology, Clinical College of \\ Chinese and Western Medicine, Anhui University of Chinese Medicine, Hefei, Anhui 230032; \\ ${ }^{3}$ Department of Natural Chemistry, School of Pharmacy, Anhui University \\ of Chinese Medicine, Hefei, Anhui 230012, P.R. China
}

Received January 29, 2016; Accepted March 6, 2017

DOI: $10.3892 /$ etm.2017.4625

\begin{abstract}
The present study aimed to explore the treatment effects of Taohongsiwu decoction on the liver fibrosis in a rat model, as few prior studies have investigated the anti-fibrotic effects of Taohongsiwu decoction. High-performance liquid chromatography was used to measure the concentration of ferulic acid and hydrosafflower flavin A in the decoction. Male Sprague-Dawley rats were randomly divided into control, model, colchicine (positive group), Taohongsiwu-high, Taohongsiwu-moderate and Taohongsiwu-low groups; $50 \%$ carbon tetrachloride $\left(\mathrm{CCl}_{4}\right)$ of peanut oil solution was subcutaneously injected to the rats except for the control group, and the drugs were intragastrically administered $(10 \mathrm{ml} / \mathrm{kg})$ starting at week 7 for 6 continuous weeks. The rats were deprived of food but not water for $12 \mathrm{~h}$ following the final administration, then blood was collected from the abdominal aorta. The liver tissues were obtained, fixed with $10 \%$ neutral formaldehyde, and embedded with paraffin. The concentrations of ferulic acid and hydroxysafflor flavin A in Taohongsiwu decoction were 0.12 and $0.57 \mathrm{mg} / \mathrm{ml}$, respectively. The three groups treated with Taohongsiwu decoction were found with significantly lower serum levels of aspartate aminotransferase, alanine amino transferase, collagen IV and hyaluronic acid, as well as higher level of albumin (ALB); in addition, the expression levels of $\alpha$-smooth muscle actin ( $\alpha$-SMA) and transforming growth factor- $\beta 1$ (TGF- $\beta 1$ ) mRNA and collagen I were significantly downregulated. Taohongsiwu decoction improved the liver function, reduced the collagen deposition in the serum and liver tissues, and inhibited the expression of $\alpha$-SMA and TGF- $\beta 1$.
\end{abstract}

Correspondence to: Dr Jun Li, School of Pharmacy, Anhui Medical University, 103 Meishan Road, Hefei, Anhui 230032, P.R. China

E-mail: lijun@ahmu.edu.cn

Key words: Taohongsiwu decoction, hepatic cirrhosis, $\alpha$-smooth muscle actin, collagen I, transforming growth factor- $\beta 1$

\section{Introduction}

Hepatic fibrosis (HF) is a serious health problem worldwide. The deregulated repairing reaction of liver tissues after chronic hepatic injuries is the pathological basis of HF. The continuous damage of liver tissue due to HF may ultimately result in hepatic cirrhosis, which is a major causes of liver disease-associated mortality (1). Using an aggressive method to treat the primary disease and HF is effective in preventing and even reversing $\mathrm{HF}$ and hepatic cirrhosis (2). Taohongsiwu decoction, is a traditional Chinese medicinal recipe that contains multiple Chinese herbs. For instance, peach keruel (3) contains amygdalin, and thus could inhibit the synthesis (4) and secretion of collagens I, III, and IV, as well as laminin. Carthamus tinctorius and hydroxysafflor flavin A have been suggested to inhibit the activation and transition of hepatic stellate cells (HSCs) (5), and their anti-lipid peroxidation effects could also reduce the degree of hepatocyte swelling and cirrhosis, thus improving the repair of damaged liver tissues (6). Herbs in the genus Angelica (7) are purported to inhibit the proliferation of fibroblasts, reduce the synthesis of collagens, and protect the rat models of HF induced by carbon tetrachloride $\left(\mathrm{CCl}_{4}\right)$. Total glucosides of peony (8) may inhibit the expression of nuclear factor $\kappa \mathrm{B}$ and transforming growth factor- $\beta 1$ (TGF- $\beta 1$ ) in the fibrotic liver tissues of rats. Ligustrazine (tetramethylpyrazine) (9) could inhibit the TGF- $\beta 1$-induced expression of connective tissue growth factor gene, thus blocking the synthesis of collagen I and resulting in anti-fibrosis effects in the liver. However, the mechanisms involved in the anti-HF effects of Taohongsiwu decoction are still unclear. In the present study, rat models of $\mathrm{HF}$ induced by $\mathrm{CCl}_{4}$ were used to comprehensively investigate the anti-HF effects of Taohongsiwu decoction and the underlying mechanisms, and provide evidence for the clinical application of Taohongsiwu decoction.

\section{Materials and methods}

Animals. Healthy male Sprague-Dawley rats (specific pathogen-free grade) with the body weight of 200-220 g were 
obtained from the Anhui Experimental Animal Center [SCXK (Wan) 2011-002]. The rats were acclimated for 2 weeks before the experiments.

Reagents. Taohongsiwu decoction contains peach keruel (9 g), Carthamus tinctorius (6 g), Ligusticum wallichii (6 g), radix Rehmanniae preparata (12 g), Angelica (9 g), and radix Paeoniae alba $(9 \mathrm{~g})$. All these drugs were purchased from the Anhui Xiehecheng Pharmaceutical Co., Ltd. (Bozhou, China). The catalogue numbers of the drugs were as follows: No. 13121001 for peach keruel, no. 13091001 for Carthamus tinctorius, no. 1310702 for Ligusticum wallichii, no. 13120101 for radix Rehmanniae preparata, no. 14021101 for Angelica, and no. 13072001 for radix Paeoniae alba. Ferulic acid (no. 110773-201313) and hydroxysafflor yellow (no. A111637-200906) were purchased from the Chinese National Institutes for Food and Drug Control (Beijing, China). $\mathrm{CCl}_{4}$ (analytically pure grade, no. 20091216) was obtained from Sinopharm Chemical Reagent Co., Ltd. (Shanghai, China). Lu Hua 5S Pressing First-Class peanut oil (no. 20130521) was obtained from the Shandong Luhua Group, Co., Ltd. (Shandong, China). Colchicine tablet (no. 130511) was obtained from Xishuangbanna Banna Pharmaceutical Co., Ltd. (Jinghong, China). Aspartate aminotransferase (AST; no. C010-1), alanine aminotransferase (ALT; no. C009-1), and ALB (no. A028-1) were obtained from Nanjing Jiancheng Biological technology Co., Ltd. (Nanjing, China). Hyaluronic acid (HA), Laminin protein (LN), procollagen III (PCIII) and collagen IV (IV-C) radioimmunoassay kits (no. 20140507) were obtained from the Beijing Sinouk Institute of Biological Technology (Beijing, China). A Masson staining kit (no. MST-8003/8004) was provided by Maixin Biological Technology Development Co., Ltd. (Fuzhou, China). An electrochemiluminescence (ECL) kit (no.NL178395) was provided by Thermo Fisher Scientific, Inc. (Waltham, MA, USA). Actin (no. 140829), goat-anti-mouse $\operatorname{IgG}$ (no. 112971) and goat-anti-rabbit IgG (no. 107015) were obtained from Beijing Zhongshan Jinqiao Biotechnology Co., Ltd. (Beijing, China). Primers were synthesized by Invitrogen (Thermo Fisher Scientific, Inc., Carlsbad, CA, USA). The primer sequences for the fluorescent quantitation of the rat $\beta$-actin were as follows: Forward, 5'-CCCATCTATGAGGGTTACGC-3' and reverse, 5'-TTTAATGTCACGCACGATTTC-3', and the length of the product was $150 \mathrm{bp}$. The primer sequences for the rat TGF- $\beta 1$ were as follows: Forward, 5'-CAGAGAAGAACTGCTGTG TACGG-3' and reverse, 5'-CAGACAGAAGTTGGCATGGTA GC-3', and the length of the product was $104 \mathrm{bp}$. A RevertAid first strand cDNA synthesis kit (no. 00145205) was obtained from Thermo Fisher Scientific, Inc.

Equipment. High-performance liquid chromatography (HPLC; Agilent Technologies, Santa Clara, CA, USA) was used in the present study. An ultraviolet spectrophotometer (UV-1800) was bought from the Shanghai Jinhua Scientific Instruments Co.,Ltd. (Shanghai, China). A full-electrodynamic advanced upright microscope was brought from the Olympus Corporation (Tokyo, Japan). A $\gamma$-911 full-automatic radioimmunity analyzer was brought from the China University of Science and Technology Industry Corporation. An EPS 300 electrophoresis apparatus was brought from the Tanon Science
\& Technology Co., Ltd. (Shanghai, China). A fluorescent quantitative polymerase chain reaction (PCR) system (PIKOREAL 96) was brought from the Thermo Fisher Scientific, Inc.

Preparation and measurement of Taohongsiwu decoction. Appropriate volumes of the drugs were obtained and immersed in two volumes of water for $30 \mathrm{~min}$. Then, 10 volumes of water were added, boiled and then filtered. Eight volumes of water were added to the residues, boiled and filtered. The liquid obtained by both the filtration were combined and concentrated to a concentration of $1 \mathrm{~g} / \mathrm{ml}$ crude drug. Exactly $5 \mathrm{ml}$ of the extraction was added to an evaporating dish, evaporated dry, and then $50 \%$ methanol was added to obtain a final volume of $10 \mathrm{ml}$. The liquid was mixed then filtered, and the filtered liquid was further filtered through a $0.45-\mu \mathrm{m}$ microfiltration membrane to obtain the solution for the experiment. Gradient elution was performed with the mobile phase of acetonitrile (A) and $0.1 \%$ phosphoric acid solution (B). The flow velocity used was $1.0 \mathrm{ml} / \mathrm{min}$, the column temperature was $40^{\circ} \mathrm{C}$, the sample size was $20 \mu \mathrm{l}$ and the determine wavelengths were as follows: $0-11 \mathrm{~min}, 290 \mathrm{~nm}$; 11-27 min, $320 \mathrm{~nm}$; $27-45 \mathrm{~min}, 230 \mathrm{~nm}$; and 45-80 min, $270 \mathrm{~nm}$. An HPLC instrument (Agilent Technologies) was used for measuring the concentration of the liquid (Figs. 1 and 2).

Inducing ratmodel of $H F(10)$. Male Sprague-Dawley rats with the body weight of 200-220 g were obtained, and $50 \% \mathrm{CCl}_{4}$ of peanut oil solution $(0.1 \mathrm{ml} / \mathrm{kg}$, freshly prepared) was subcutaneously injected to the back of the rats (twice/week) for 12 continuous weeks to induce the rat model of HF.

Grouping and drug administration. Rats were randomly divided into model, colchicine $(2 \mathrm{mg} / \mathrm{kg})$, Taohongsiwu-high $(17 \mathrm{~g} / \mathrm{kg})$, Taohongsiwu-moderate $(8.5 \mathrm{~g} / \mathrm{kg})$, and Taohongsiwu-low $(4.25 \mathrm{~g} / \mathrm{kg})$ groups with 10 rats in each group. Another 10 rats were included as the control group. Since week 7 after the model induction, the intragastric administration of the drugs was performed with the dose of $10 \mathrm{ml} / \mathrm{kg}$ (once per day) for 6 continuous weeks, while the same volume of distilled water was administered for the rats in the model and control groups. The rats were deprived of food but not water for $12 \mathrm{~h}$ after the last drug administration; chloral hydrate $(300 \mathrm{mg} / \mathrm{kg}$ body weight) was used to anesthetize the rats. Then, blood was collected from the abdominal aorta, and serum was separated. Part of the liver of the rats was also collected to measure the parameters.

Measuring serum indexes. Blood was collected from the abdominal aorta of the rat, placed at room temperature for $1 \mathrm{~h}$, then centrifuged at $825 \mathrm{x} \mathrm{g}$ for $10 \mathrm{~min}$ to separate the serum. The serum level of ALB and activities of AST and ALT were measured by colorimetry, and serum levels of HA, LN, PCIII and IV-C were measured by radioimmunoassay.

Pathological examinations of the liver tissues. For each rat, part of the liver was obtained, fixed with $10 \%$ formalin, embedded with paraffin, and sliced. Then, hematoxylin and eosin (H\&E) and Masson staining were performed for pathological examinations. 


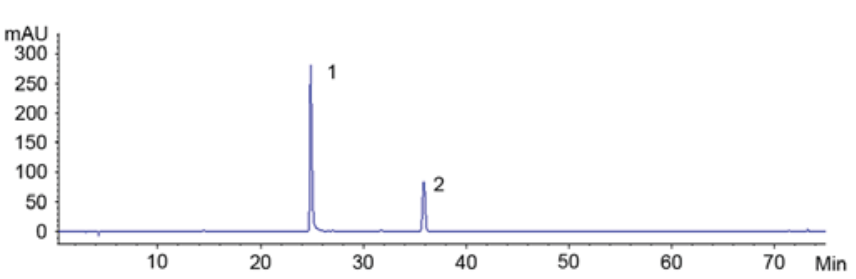

Figure 1. Standard solutions (1 indicates hydrosafflow yellow A, and 2 indicates ferulic acid).

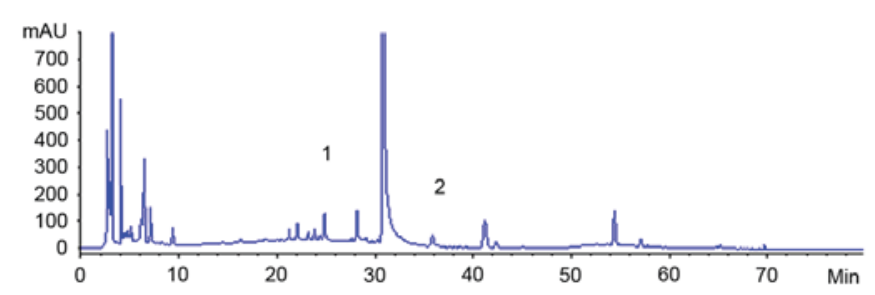

Figure 2. Water extraction of Taohongsiwu decoction (1 indicates hydrosafflow yellow $\mathrm{A}$, and 2 indicates ferulic acid).

Measuring Col I levels in the liver tissues. Col I levels in the liver tissues were measured by western blot analysis. In brief, liver tissues were collected and weighed. A total of $100 \mathrm{mg}$ liver tissue was obtained, then $1 \mathrm{ml}$ radioimmunoprecipitation assay cell lysis buffer (containing $1 \mathrm{mM}$ phenylmethylsuofonyl fluoride) was added to lyse the cells. The mixture was then centrifuged at $13,200 \mathrm{x} \mathrm{g}$ for $10 \mathrm{~min}$, and the supernatant was collected. Proteins were separated by $10 \%$ SDS-PAGE and transferred onto a nitrocellulose filter membrane (Merck KGaA, Darmstadt, Germany). Then the protein-loading membrane was immediately placed into the western washing buffer for $5 \mathrm{~min}$ to remove the transmembrane buffer. The western blocking buffer (5\% skim milk powder) was then added, and the membrane was blocked at room temperature for $2 \mathrm{~min}$ with gentle shake. Primary rabbit antibody for Collagen I (1:500, 8\% separation gel) was added, the membrane was incubated at $4^{\circ} \mathrm{C}$ overnight with gentle shaking. Then Tris-Buffered Saline and Tween 20 (TBST) was used to wash the membrane three times for $5 \mathrm{~min}$ each time. The horseradish peroxidase-labeled secondary antibody $(1: 10,000)$ was added, the membrane was blocked at room temperature for $2 \mathrm{~h}$; then washing buffer (TBST) was used to wash the membrane three times for $10 \mathrm{~min}$ each time. The ECL kit was used to measure the protein level. Image $\mathrm{J}$ software (National Institutes of Health, USA) was used to analyze the gray values.

Measuring $\alpha$-SAM expression in the liver tissues. Immunohistochemistry was used to measure the $\alpha$-SMA expression in the liver tissues. In brief, the liver tissues were embedded with paraffin, continuously sliced (thickness, $5 \mu \mathrm{m}$ ) and deparaffinated with xylene. Then, gradient ethanol hydration was performed, and the slices were washed with distilled water and incubated in $3 \% \mathrm{H}_{2} \mathrm{O}_{2}$ deionized water mixture for $15 \mathrm{~min}$ to end the activity of endogenous peroxidase. Phosphate buffered saline (PBS; $\mathrm{pH}$ 7.4) was used to wash the slices three times for $3 \mathrm{~min}$ each time, then normal goat serum (Boster Biological Technology, Pleasanton CA, USA) working buffer used as a blocking solution was added and incubated for $15 \mathrm{~min}$. The liquid was discarded, and the slices were not washed. The appropriately diluted primary antibody was added, and the slices were incubated at $4^{\circ} \mathrm{C}$ overnight. Next, the slices were washed again with PBS (pH 7.4) three times for 5 min each time, after which biotinylated secondary antibody was added, incubated at $37^{\circ} \mathrm{C}$ for $15 \mathrm{~min}$, washed with PBS (pH 7.4) three times for 5 min each time, developed with diaminobenzidine for $2 \mathrm{~min}$, and then water was added to incubate for $5 \mathrm{~min}$ to end the reaction. The slices were blotted dry and mounted with neutral balsam. The light microscope was used for the observation, under which $\alpha$-SMA-positive cells appeared brown yellow. Six slices were selected from each group, and at least five non-overlapping, complete visual fields were randomly selected in each slice under high magnitude. Image-Pro Plus 6.0 software (Media Cybernetics, Inc., Silver Spring, MD, USA) was used to measure the average optical density and the integrated optical density in each visual field, and the mean values were calculated for the statistical analysis.

Measuring TGF- $\beta 1$ mRNA expression in the liver tissues. Fluorogenic quantitative PCR was used to measure the TGF- $\beta 1$ mRNA expression in the liver tissues. The processes were as follows. A total of 50-100 mg liver tissues were obtained, cut into small pieces, ground in liquid nitrogen, and then $1 \mathrm{ml}$ TRIzol was added and homogenized. Then the homogenate was centrifuged at $4^{\circ} \mathrm{C}, 13,200 \mathrm{x} \mathrm{g}$ for $10 \mathrm{~min}$ to remove the insufficiently lysed tissues and fat. Chloroform $(0.2 \mathrm{ml})$ was added, violently mixed for $15 \mathrm{sec}$, and placed at room temperature for $3 \mathrm{~min}$. Then another centrifugation at $4^{\circ} \mathrm{C}, 13,200 \mathrm{x} \mathrm{g}$ was performed for $15 \mathrm{~min}$, and the supernatant $(\sim 500 \mu \mathrm{l})$ was transferred to another Eppendorf (EP) tube. Isopropanol $(0.5 \mathrm{ml})$ was added, mixed gently, placed at room temperature for $10 \mathrm{~min}$, centrifuged at $4^{\circ} \mathrm{C}, 13,200 \mathrm{xg}$ for $10 \mathrm{~min}$, and the supernatant was discarded. A total of $1 \mathrm{ml}$ of $75 \%$ ethanol [prepared with diethylpyrocarbonate (DEPC) water] was then added, after which the mixture was centrifuged at $4^{\circ} \mathrm{C}, 13,200 \mathrm{x}$ g for $5 \mathrm{~min}$, and the supernatant was discarded. Then the EP tube was placed at room temperature for 30 min to allow the RNA dry. DEPC (20-50 $\mu \mathrm{l})$ water was added, heated to $55^{\circ} \mathrm{C}$ to help dissolve the RNA, and then stored at $-80^{\circ} \mathrm{C}$ until use.

Fluorogenic quantitative PCR analysis. Total RNA (3 $\mu \mathrm{g})$ and $1 \mu \mathrm{l}$ of $10 \mu \mathrm{M}$ oligo (3dT) were added into a $0.2-\mathrm{ml}$ EP tube, and then DEPC water was added to obtain a final volume of $12 \mu 1$. The tube was mixed gently and centrifuged transiently. The tubes were heated to $65^{\circ} \mathrm{C}$ for $5 \mathrm{~min}$ on the PCR instrument, and then placed in an ice bath for $3 \mathrm{~min}$. A total of $4.0 \mu \mathrm{l}$ of $5 \mathrm{X}$ reaction buffer, $2 \mu \mathrm{l}$ of $10 \mathrm{mM}$ dNTP mix, $1 \mu \mathrm{l}$ of RNase inhibitor, and $1 \mathrm{nl}$ of RevertAid M-MuLV Reverse Transcriptase were added into each EP tube for the reaction (at $42^{\circ} \mathrm{C}$ for $60 \mathrm{~min}$ and then at $70^{\circ} \mathrm{C}$ for $5 \mathrm{~min}$ ). The reaction liquid was the cDNA, and was stored at $-80^{\circ} \mathrm{C}$ until use.

The reaction system was as follows: cDNA template, $1 \mu \mathrm{l}$; RNase free water, $2 \mu \mathrm{l}$; forward and reverse primers $(10 \mu \mathrm{M})$, $1 \mu \mathrm{l}$ each; and $2 \mathrm{X}$ SYBR Green mixture, $5 \mu \mathrm{l}$. The reaction conditions were as follows: $95^{\circ} \mathrm{C}$ for $5 \mathrm{~min}, 95^{\circ} \mathrm{C}$ for $10 \mathrm{sec}$, and $60^{\circ} \mathrm{C}$ for $30 \mathrm{sec}$ for 40 cycles. A relative quantification 
Table I. Effects of Taohongsiwu decoction on the serum levels of AST, ALT and ALB of the rat models of hepatic fibrosis.

\begin{tabular}{lrrrr}
\hline Group & N & AST $(\mathrm{U} / \mathrm{l})$ & ALT $(\mathrm{U} / \mathrm{l})$ & ALB $(\mathrm{g} / \mathrm{l})$ \\
\hline Control & 10 & $13.91 \pm 5.01^{\mathrm{a}}$ & $12.69 \pm 5.35^{\mathrm{a}}$ & $31.93 \pm 3.59$ \\
Model & 9 & $48.42 \pm 7.65^{\mathrm{b}}$ & $83.31 \pm 13.85^{\mathrm{b}}$ & $26.17 \pm 2.44^{\mathrm{c}}$ \\
Positive & 8 & $37.78 \pm 7.71^{\mathrm{b}, \mathrm{d}}$ & $71.97 \pm 11.66^{\mathrm{b}}$ & $26.43 \pm 2.56$ \\
Taohongsiwu & & & & \\
Low & 9 & $43.49 \pm 9.94^{\mathrm{b}}$ & $72.23 \pm 14.05^{\mathrm{b}}$ & $24.77 \pm 3.69$ \\
Moderate & 9 & $37.01 \pm 8.05^{\mathrm{b}, \mathrm{d}}$ & $66.53 \pm 13.39^{\mathrm{b}}$ & $29.40 \pm 1.54^{\mathrm{d}}$ \\
High & 10 & $22.48 \pm 6.62^{\mathrm{a}, \mathrm{d}}$ & $51.16 \pm 10.73^{\mathrm{b}, \mathrm{d}}$ & $28.99 \pm 2.79$ \\
\end{tabular}

${ }^{\mathrm{a}} \mathrm{P}<0.01$ vs. model group; ${ }^{\mathrm{b}} \mathrm{P}<0.01$ vs. control group; ${ }^{\mathrm{C}} \mathrm{P}<0.05$ vs. control group; ${ }^{\mathrm{d}} \mathrm{P}<0.05$ vs. model group. AST, aspartate aminotransferase; ALT, alanine aminotransferase; ALB, albumin.

method was used for analysis, and the index used for the analysis was $2^{-\Delta \Delta \mathrm{Cq}}$.

Statistical analysis. SPSS 11.0 software (SPSS, Inc., Chicago, IL, USA) was used for the statistical analysis. All the data are presented as the mean \pm standard deviation, and compared with one-way analysis of variance followed by least-significant difference t-test.

\section{Results}

Concentrations of hydrosafflow yellow and ferulic acid in Taohongsiwu decoction. HPLC examinations showed that the concentration of ferulic acid and hydrosafflow yellow in Taohongsiwu decoction was 0.12 and $0.57 \mathrm{mg} / \mathrm{ml}$, respectively.

Effects of Taohongsiwu decoction on the serum levels of AST, $A L T$, and $A L B$ of the rat models of $H F$. As shown in Table I and Fig. 3, the AST and ALT levels increased significantly in all the groups of rat models, while the ALB level decreased significantly compared with the control group. After the drugs were administered for 6 continuous weeks, the AST level in the colchicine, Taohongsiwu-high, and Taohongsiwu-moderate groups decreased significantly, and the differences with the model group were statistically significant; while the ALB level in the Taohongsiwu-moderate group increased significantly, and the difference with the model group was statistically significant.

Effects of Taohongsiwu decoction on the serum levels of $L N$, PIII, IV-C and HA of the rat models of HF. As shown in Fig. 4, the serum levels of LN, PCIII, IV-C and HA increased in all the groups of rat models, among which the increase of the IV-C and HA levels was statistically significant compared with the control group $(\mathrm{P}<0.05)$. The levels of all the four indexes improved after the drug administration, among which the IV-C and HA levels in the Taohongsiwu-high group, and the IV-C level in the Taohongsiwu-moderate group decreased significantly, and the differences with the model group were statistically significant $(\mathrm{P}<0.05)$.

Effects of Taohongsiwu decoction on the pathological changes of the liver tissues of the rat models of HF. The

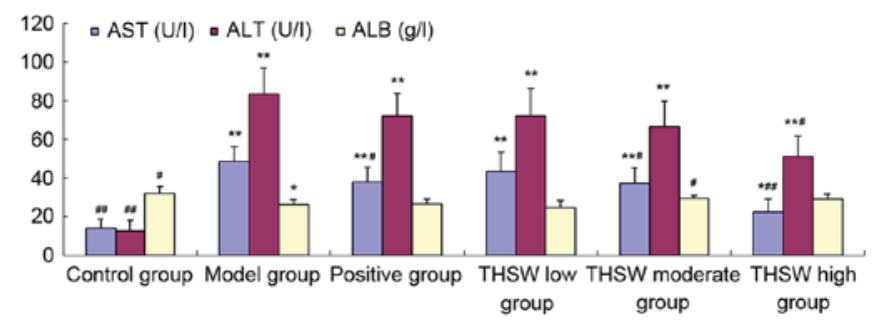

Figure 3. Effects of Taohongsiwu decoction on the serum levels of AST, ALT, and ALB of the rat models of hepatic fibrosis. ${ }^{*} \mathrm{P}<0.05,{ }^{* *} \mathrm{P}<0.01$ vs. control group; ${ }^{\#} \mathrm{P}<0.05,{ }^{\#} \mathrm{P}<0.01$, vs. model group. AST, aspartate aminotransferase; ALT, alanine aminotransferase; ALB, albumin; THSW, Taohongsiwu.

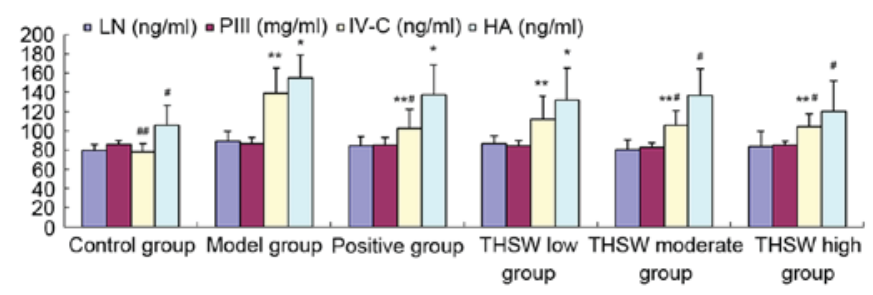

Figure 4. Effects of Taohongsiwu decoction on the serum levels of LN, PIII, IV-C, and HA of the rat models of hepatic fibrosis. ${ }^{*} \mathrm{P}<0.05,{ }^{* *} \mathrm{P}<0.01 \mathrm{vs}$. control group; ${ }^{\#} \mathrm{P}<0.05,{ }^{\# \#} \mathrm{P}<0.01$, vs. model group. LN, laminin; PIII, procollagen III; IV-C, procollagen IV; HA, hyaluronic acid; THSW, Taohongsiwu.

effects of Taohongsiwu decoction on the pathological changes of the liver tissues of the rat models of HF are shown in Figs. 5 and $6 . H \& E$ staining showed that the hepatic lobules in the control group were with complete structure, and the hepatic cords arranged regularly; while no inflammatory cell infiltration in the portal area of the hepatic lobules or fibrotic tissue hyperplasia was found. For the rats in the model group, the structure of the hepatic lobules were found damaged, the arrangement of the hepatic cords was irregular, the hepatic cells were with diffused vacuolar degenerations and necrosis. The portal area expanded and presented with large amounts of fibrotic tissue hyperplasia, which separated the hepatic lobules into several hepatocyte clusters of different sizes. The damages of the hepatic lobules in the control group and the groups treated with drugs were evidently decreased, with limited inflammatory cell infiltration in the liver tissues 

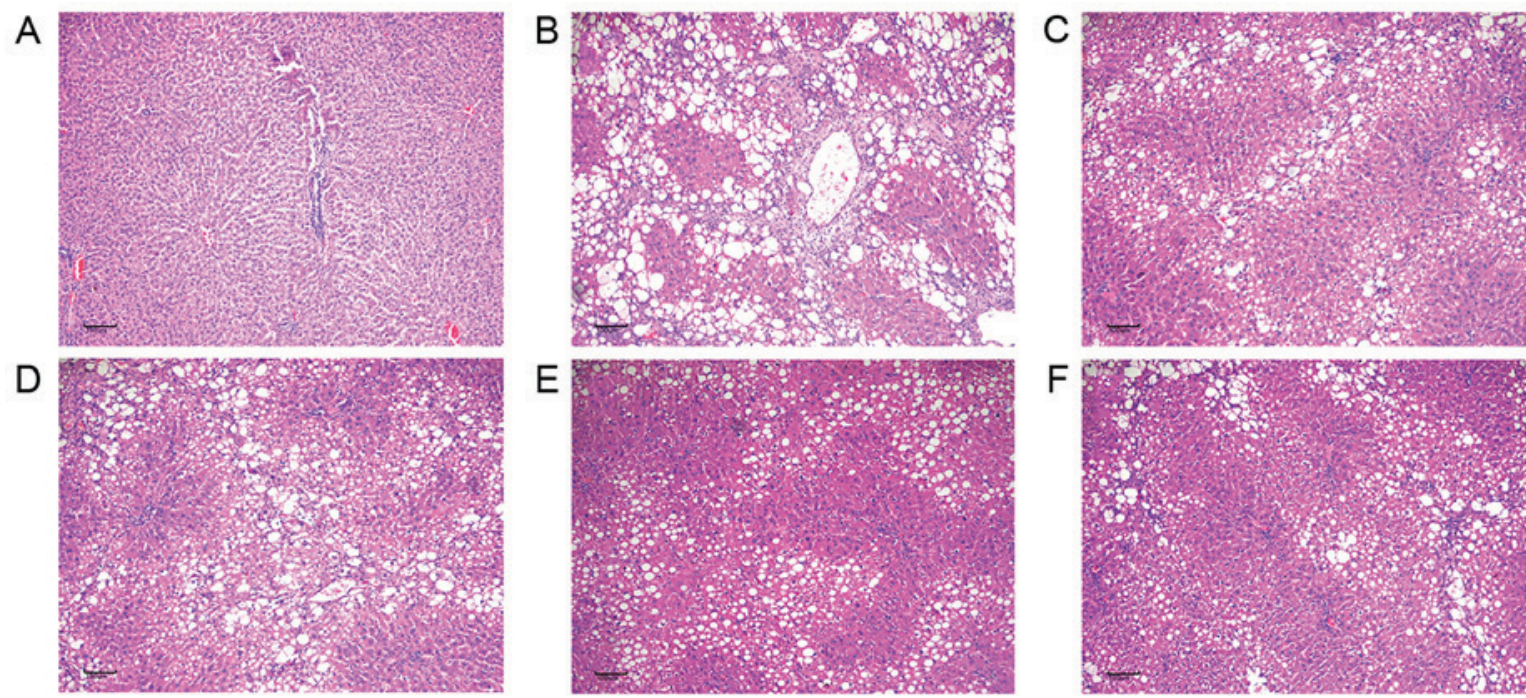

Figure 5. Effects of Taohongsiwu decoction on the liver tissues of the rat models of hepatic fibrosis (hematoxylin and eosin; magnification, x100). (A) Control group; (B) model group; (C) positive group; (D) Taohongsiwu-low group; (E) Taohongsiwu-moderate group; and (F) Taohongsiwu-high group.
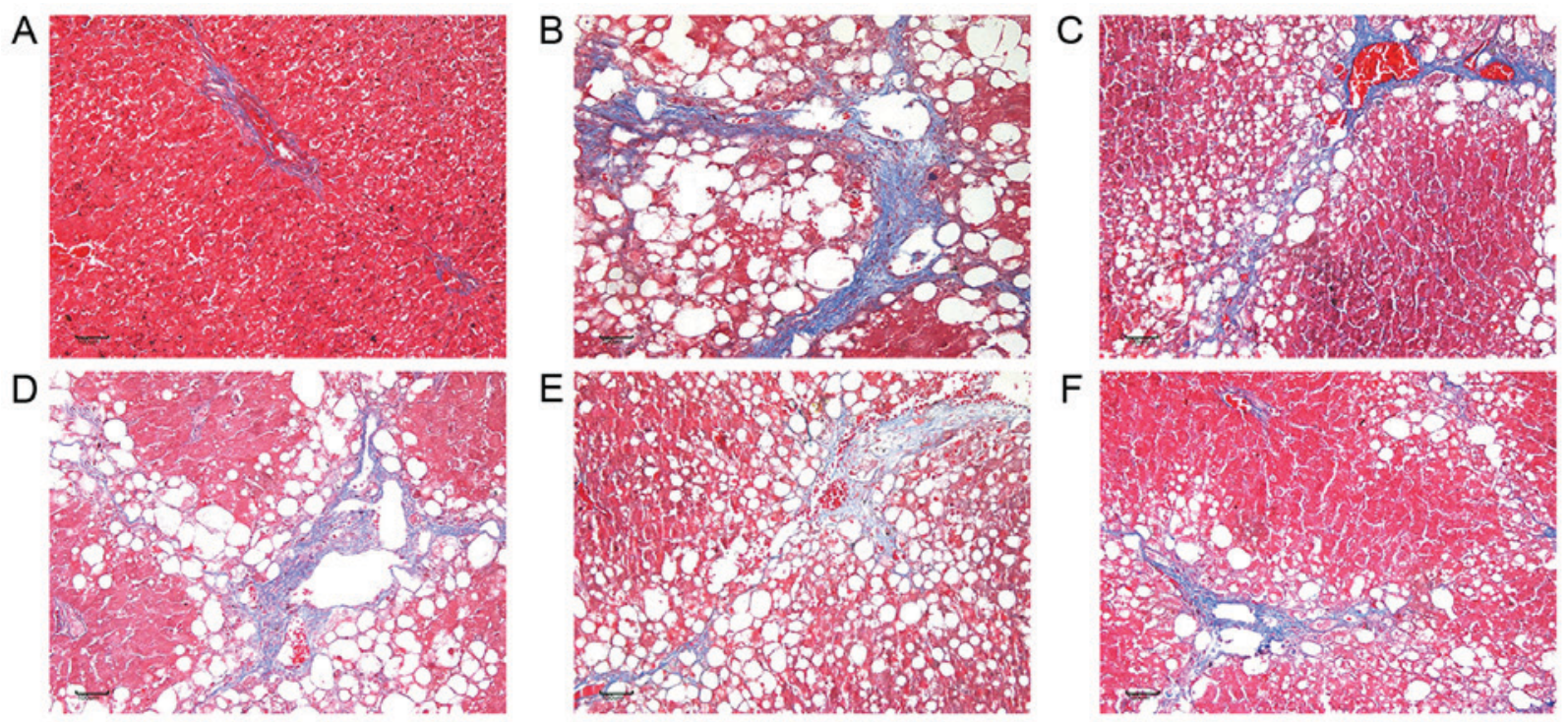

Figure 6. Effects of Taohongsiwu decoction on the liver tissues of the rat models of hepatic fibrosis (Masson; magnification, x200). (A) Control group; (B) model group; (C) positive group; (D) Taohongsiwu-low group; (E) Taohongsiwu-moderate group; and (F) Taohongsiwu-high group.

observed. Furthermore, the swelling of the liver cells and the degree of fatty degeneration were evidently lesser than in the model group.

Masson staining showed that the liver tissues in the control group presented with limited collagen deposition at the venous walls and the bile duct walls in the portal area. For the rats in the model group, extensive fibrotic tissue hyperplasia was found in the interstitial tissues; the collagens increased and enlarged, and diffused in the liver tissues to separate the hepatic lobules into several pseudolobules of different sizes. While the collagens in the liver tissues of the rats treated with Taohongsiwu decoction were evidently decreased, the fibrous septum was short and narrow, and no pseudolobule was observed.

Effects of Taohongsiwu decoction on the Col I level in the liver tissues of the rat models of HF. As shown in Fig. 7,

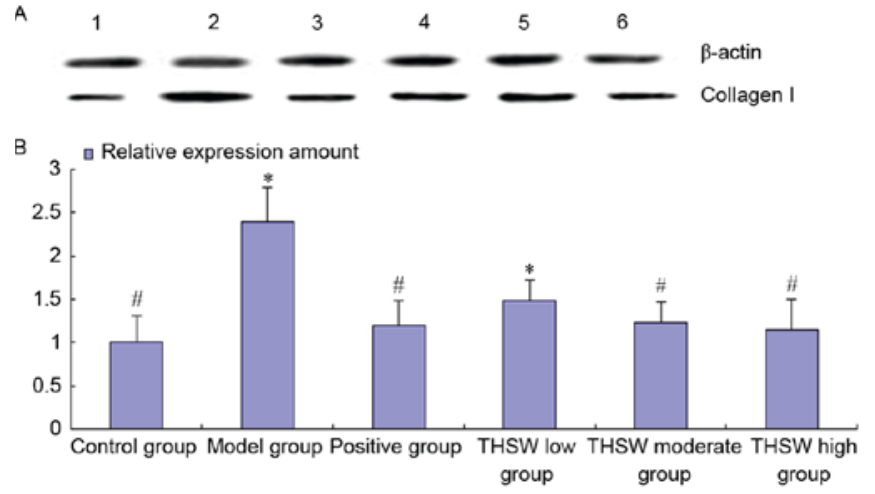

Figure 7. (A) 1, Control group; 2, model group; 3, positive group; 4 , Taohongsiwu-low group; 5, Taohongsiwu-moderate group; and 6 , Taohongsiwu-high group. (B) Effects of Taohongsiwu decoction on the collagen I level in the liver tissues of the rat models of hepatic fibrosis $(n=3)$. "P<0.05 vs. control group; ${ }^{"} \mathrm{P}<0.05$ vs. model group. THSW, Taohongsiwu. 
A

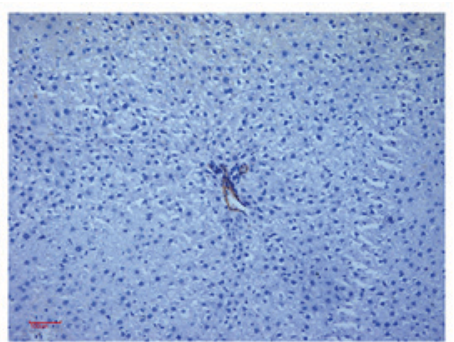

D

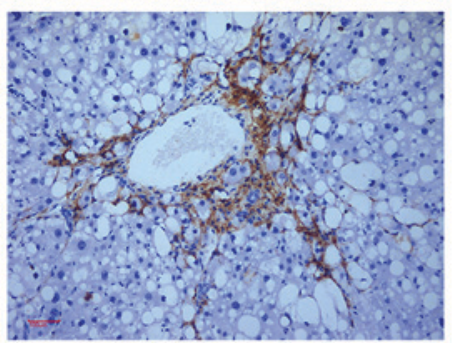

B

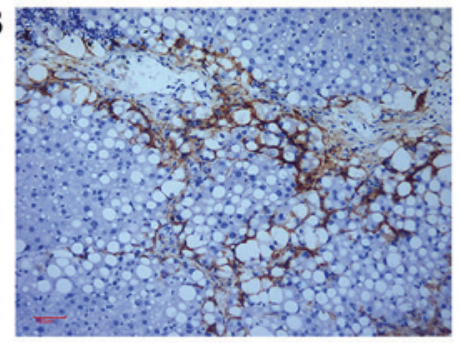

$\mathrm{E}$

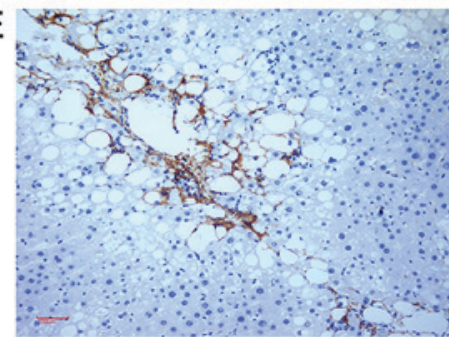

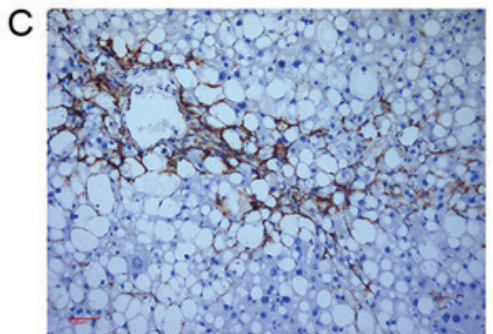

$\mathrm{F}$

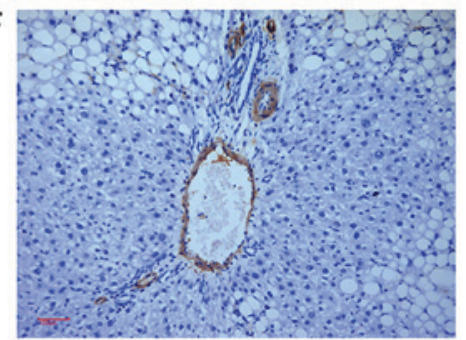

Figure 8. Effects of Taohongsiwu decoction on $\alpha$-smooth muscle actin expression in the liver tissues of the rat models of hepatic fibrosis (magnification, $\mathrm{x} 200$ ). (A) Control group; (B) model group; (C) positive group; (D) Taohongsiwu-low group; (E) Taohongsiwu-moderate group; and (F) Taohongsiwu-high group.

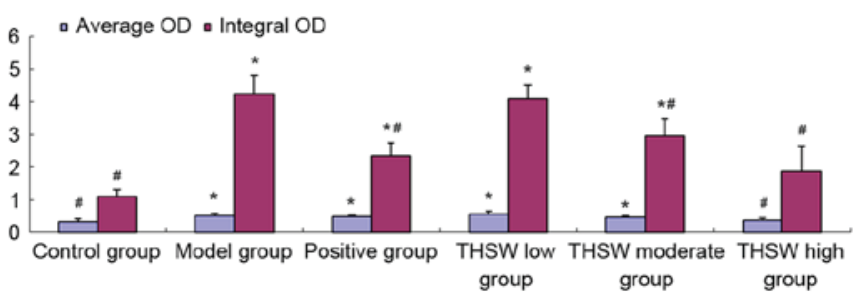

Figure 9. Effects of Taohongsiwu decoction on $\alpha$-smooth muscle actin expression in the liver tissues of the rat models of hepatic fibrosis $(n=6)$. ${ }^{*} \mathrm{P}<0.05$ vs. control group; ${ }^{\text {"P}} \mathrm{P}<0.05$ vs. model group. THSW, Taohongsiwu.

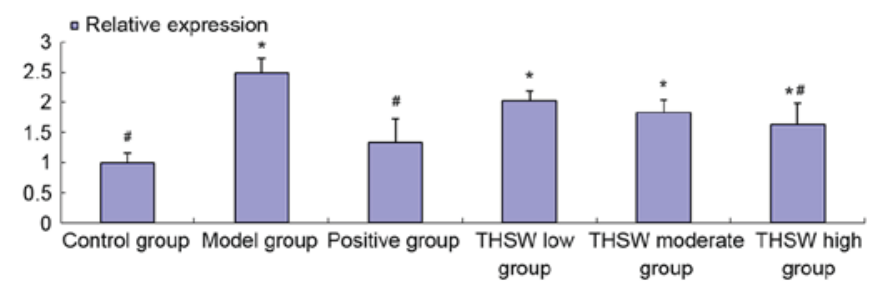

Figure 10. Effects of Taohongsiwu decoction on transforming growth factor- $\beta 1$ mRNA expression in the liver tissues of the rat models of hepatic fibrosis $(n=5) . ~{ }^{*} \mathrm{P}<0.05$ vs. control group; ${ }^{~} \mathrm{P}<0.05$ vs. model group. THSW, Taohongsiwu.

the collagen level in the liver tissues increased in all the rats at 12 weeks after the model induction, and the level in the model and Taohongsiwu-low groups was significantly different from the control group. After treatment for 6 weeks, the collagen level in the liver tissues of the rats decreased, and the collagen level in the positive, Taohongsiwu-high, and Taohongsiwu-moderate groups was significantly reduced compared with the model group $(\mathrm{P}<0.05)$.

Effects of Taohongsiwu decoction on $\alpha$-SMA expression in the liver tissues of the rat models of HF. As shown in Figs. 8 and 9, $\alpha$-SMA-positive tissues in the liver tissues are shown as brown yellow. Limited $\alpha$-SMA expression was detected at the vascular walls of the liver tissues in the control group. For the rats in the model group, the expression of $\alpha$-SMA was found not only at the vascular walls, but also widely spread at the portal area, fibrous septum, and the adjacent hepatic sinusoids. After treated with the drugs for six continuous weeks, the expression of $\alpha$-SMA in the liver tissues decreased significantly, especially in the positive, Taohongsiwu-high, and Taohongsiwu-moderate groups; the $\alpha$-SMA expression level in these three groups was evidently lower compared with the model group (Fig. 9).

Effects of Taohongsiwu decoction on TGF- $\beta 1$ mRNA expression in the liver tissues of the rat models of HF. As shown in Fig. 10, the expression of TGF- $\beta 1$ mRNA increased significantly in the all the model groups, and the difference with the control group was statistically significant $(\mathrm{P}<0.05)$. After treatment with the drugs for six continuous weeks, the TGF- $\beta 1$ mRNA expression decreased, particularly in the colchicine and Taohongsiwu-high groups, in which the expression was significantly decreased compared with the model group $(\mathrm{P}<0.05)$.

\section{Discussion}

$\mathrm{HF}$ is an inevitable stage of the progression of chronic liver diseases into cirrhosis, and is caused by the imbalanced proliferation and degeneration of fibrosis, which could cause the overdeposition of fibrillar connective tissues in the liver (11). Currently, no specific drug that could cure liver cirrhosis is available. However, previous results have shown that HF is reversible, and preventing the deposition and promoting the degeneration of extracellular matrix is one of the effective methods in anti-fibrosis and treating liver cirrhosis (12).

The development and progression of HF is a complex systemic pathophysiological process, which involves multiple cytokines and the network of intracellular signaling molecules. The activation of HSC has been acknowledged as the central factor in developing HF (13). When the hepatocytes are damaged by ethanol, viral infection, or drugs, the hepatocytes 
occur the inflammatory reaction and stimulate the secretion of cytokines (such as TGF- $\beta 1$, PDGF and VEGF) by Kupffer cells, liver sinusoidal endothelial cells and macrophages will occur (14). The stimuli will cause phenotype transformation of the HSC from quiescent phenotype to myofibroblast (MFB)-like phenotype that express $\alpha$-SMA, which could be further activated and transformed to MFB. MFB phenotype HSC to secrete large quantities of extracellular matrix including collagen I and III, HA, and laminin; reduce the activity of collagenase and the degeneration of collagen, resulting in the imbalance of the production and degeneration of extracellular matrix, formation of fibrosis, and a large expression of $\alpha$-SMA protein, which has been considered as an important marker of the activation of HSC (15). The activated HSC could also secrete cytokines including TGF- $\beta 1$ and tumor necrosis factor- $\alpha$, and further promote activation of themselves. Therefore, TGF- $\beta 1$ is crucial for the activation, transformation, differentiation and regulation of HSCs. TGF- $\beta 1$ has been proposed to be a potential factor promoting the fibrosis of HSC, and the level of TGF- $\beta 1$ may indicate the degree of inflammation, necrosis and fibrosis in chronic HF tissues (16).

The mechanisms of the currently used drugs for the treatment of HF include inhibition of liver inflammation and immune response, anti-oxidative damages, inhibition of the activation of HSC, inhibition of the synthesis of extracellular matrix, and acceleration of the degeneration of extracellular matrix. Previous studies have suggested that Taohongsiwu decoction treatment may be effective against acute liver damages (17), and may inhibit the activation of HSC (18). The active ingredients in Taohongsiwu decoction include amygdalin, ligustrazine and hydrosafflow yellow that could reduce liver damages, inhibit HSC activation, improve the structure of liver tissues, inhibit the deposition of collagen and delay the progression of liver fibrosis (19-21). The findings of the present study suggest that Taohongsiwu decoction may improve the liver function of the rat models of HF, reduce the collagen deposition in the serum and liver tissues, and inhibit the expression of $\alpha$-SMA protein and TGF- $\beta 1$ mRNA. These findings suggest that Taohongsiwu decoction treatment exhibits effective protective effects against HF, and the underlying mechanisms could be associated with liver protection and inhibition of TGF- $\beta$ and HSC activation. Further studies are needed to further investigate the precise underlying mechanisms.

\section{Acknowledgements}

The present study was supported by the Anhui Province College Students Innovative and Entrepreneurial Training Project (grant no. AH201310369054).

\section{References}

1. Imani F, Motavaf M, Safari S and Alavian SM: The therapeutic use of analgesics in patients with liver cirrhosis: A literature review and evidence-based recommendations. Hepat Mon 14: e23539, 2014.

2. Sun M and Kisseleva T: Reversibility of liver fibrosis. Clin Res Hepatol Gastroenterol 39 (Suppl 1): 60-63, 2015.
3. Xu LM, Liu P, Liu C, Hong JH, Lu G, Xue HM, Zhu JL and Hu YY: Observation on the action of extractum semen Persicae on anti-fibrosis of liver. Zhongguo Zhong Yao Za Zhi 19: 491-494, 512. 1994 (In Chinese).

4. Wang YQ: Effect and molecular mechanism of peach kernel extract on liver fibrosis. J Med Theor Pract 15: 2015-2016, 2014 (In Chinese).

5. Zhang Y, Guo J, Dong H, Zhao X, Zhou L, Li X, Liu J and Niu Y: Hydroxysafflor yellow A protects against chronic carbon tetrachloride-induced liver fibrosis. Eur J Pharmacol 660: 438-444, 2011

6. Wang CY, Liu Q, Huang QX, Liu JT, He YH, Lu JJ and Bai XY: Activation of PPAR $\gamma$ is required for hydroxysafflor yellow $\mathrm{A}$ of Carthamus tinctorius to attenuate hepatic fibrosis induced by oxidative stress. Phytomedicine 20: 592-599, 2013.

7. Wang WQ, Wang BX and Shao WW: Effect of Angelica on the collagen content of hepatic tissue in liver fibrosis model rats induced by carbon tetrachloride. Hei Long Jiang Yi Yao 26: 25, 2003 (In Chinese).

8. Lu JT, Sun WY and Liu H: Effects of total glucosides of paeony on protein expression of NF-kappaB and TGF- $\beta 1$ in hepatic tissue of rats with immunological hepatic fibrosis. Chi Pharmacological Bulletin 24: 588-592, 2008.

9. Lu B, Yu L, Li S, Si S and Zeng Y: Alleviation of CCl4-induced cirrhosis in rats by tetramethylpyrazine is associated with downregulation of leptin and TGF-beta1 pathway. Drug Chem Toxicol 33: 310-315, 2010.

10. Huang Q, Li Y, Zhang S, Huang R, Zheng L, Wei L, He M, Liao M, Li L, Zhuo L and Lin X: Effect and mechanism of methyl helicterate isolated from Helicteres angustifolia (Sterculiaceae) on hepatic fibrosis induced by carbon tetrachloride in rats. J Ethnopharmacol 143: 889-895, 2012.

11. Liu T, Wang P, Cong M, Zhang D, Liu L, Li H, Zhai Q, Li Z, Jia L and You H: Matrix metalloproteinase-1 induction by diethyldithiocarbamate is regulated via Akt and ERK/miR222/ETS-1 pathway in hepatic stellate cells. Biosci Rep 36: pii: e00371, 2016.

12. Choi JH, Hwang YP, Park BH, Choi CY, Chung YC and Jeong HG: Anthocyanins isolated from the purple-fleshed sweet potato attenuate the proliferation of hepatic stellate cells by blocking the PDGF receptor. Environ Toxicol Pharmacol 31: 212-219, 2011.

13. Han M, Liu X, Liu S, Su G, Fan X, Chen J, Yuan Q and Xu G: 2,3,7,8-Tetrachlorodibenzo-p-dioxin (TCDD) induces hepatic stellate cell (HSC) activation and liver fibrosis in C57BL6 mouse via activating Akt and NF- $\mathrm{KB}$ signaling pathways. Toxicol Lett 273: 10-19, 2017.

14. Marrone G, Shah VH and Gracia-Sancho J: Sinusoidal communication in liver fibrosis and regeneration. J Hepatol 65: 608-617, 2016.

15. Li S, Wang L, Yan X, Wang Q, Tao Y, Li J, Peng Y, Liu P and Liu C: Salvianolic acid B attenuates rat hepatic fibrosis via downregulating angiotensin II signaling. Evid Based Complement Alternat Med 2012: 160726, 2012.

16. Qu Y, Zong L, Xu M, Dong Y and Lu L: Effects of $18 \alpha$-glycyrrhizin on TGF- $\beta 1 / \mathrm{Smad}$ signaling pathway in rats with carbon tetrachloride-induced liver fibrosis. Int J Clin Exp Pathol 8: 1292-1301, 2015.

17. Ju MY, Tang YZ and Ji YH: Effect of Taohong Siwu decoction on acute liver injury in model rats induced CCl4. Shandong J Tradit Chin Med: 751-752, 2012 (In Chinese).

18. Luo LQ and Chen DB: Effect of Taohong siwu decoction on the proliferation of hepatic stellate cell in rats with hepatic fibrosis. Yi Chun Xue Yuan Xue Bao 36: 69-72, 2014 (In Chinese).

19. Wu X, Zhang F, Xiong X, Lu C, Lian N, Lu Y and Zheng S: Tetramethylpyrazine reduces inflammation in liver fibrosis and inhibits inflammatory cytokine expression in hepatic stellate cells by modulating NLRP3 inflammasome pathway. IUBMB Life 67: 312-321, 2015.

20. Li XM, Hu YY and Duan XH: Uniform designed research on the active ingredients assembling of Chinese medicine prescription for anti-liver fibrosis. Zhongguo Zhong Xi Yi Jie He Za Zhi 30: 58-63, 2010 (In Chinese).

21. Jiang S, Shi Z, Li C, Ma C, Bai X and Wang C: Hydroxysafflor yellow A attenuates ischemia/reperfusion-induced liver injury by suppressing macrophage activation. Int J Clin Exp Pathol 7: 2595-2608, 2014. 